\title{
Avidity observed between a bivalent inhibitor and an enzyme monomer with a single active site
}

Shiran Lacham-Hartman ${ }^{1}$, Yulia Shmidov², Evette S. Radisky³, Ronit Bitton², David B. Lukatsky ${ }^{4}$, and Niv Papo ${ }^{1 *}$

From the ${ }^{1}$ Avram and Stella Goldstein-Goren Department of Biotechnology Engineering and the National Institute of Biotechnology in the Negev, Ben-Gurion University of the Negev, Beer-Sheva 84105, Israel; ${ }^{2}$ Deprtment of Chemical Engineering and the Ilse Katz Institute for Nanoscale Science \&Technology, BenGurion University of the Negev, Beer-Sheva 84105, Israel; ${ }^{3}$ Department of Cancer Biology, Mayo Clinic Comprehensive Cancer Center, Jacksonville, Florida 32224, USA; ${ }^{4}$ Department of Chemistry, Ben-Gurion University of the Negev, Beer-Sheva 84105, Israel;

Running title: Binding avidity for single binding sites

${ }^{*}$ To whom correspondence should be addressed: Niv Papo, Department of Biotechnology Engineering and the National Institute of Biotechnology, Ben-Gurion University of the Negev; P.O.B. 653, Beer-Sheva 84105, Israel; Tel: +972-50-2029729; email: papo@bgu.ac.il.

This manuscript includes 26 pages in total, 1 supplementary material page, and 5 figures.

The supplementary material includes 2 figures and 1 table (Fig. S1: Strategy for inserting dimeric APPI into P. pastoris; Fig. S2: Mass-spectrometry spectra; Table S1: Protein concentration determination). 


\begin{abstract}
Although myriad protein-protein interactions in nature use polyvalent binding, in which multiple ligands on one entity bind to multiple receptors on another, to date an affinity advantage of polyvalent binding has been demonstrated experimentally only in cases where the target receptor molecules are clustered prior to complex formation. Here, we demonstrate cooperativity in binding affinity (i.e., avidity) for a protein complex in which an engineered dimer of the amyloid precursor protein inhibitor (APPI), possessing two fully functional inhibitory loops, interacts with mesotrypsin, a soluble monomeric protein that does not self-associate or cluster spontaneously. We found that each inhibitory loop of the purified APPI homodimer was over three-fold more potent than the corresponding loop in the monovalent APPI inhibitor. This observation is consistent with a suggested mechanism whereby the two APPI loops in the homodimer simultaneously and reversibly bind two corresponding mesotrypsin monomers to mediate mesotrypsin dimerization. We propose a simple model for such dimerization that quantitatively explains the observed cooperativity in binding affinity. Binding cooperativity in this system reveals that the valency of ligands may affect avidity in protein-protein interactions including those of targets that are not surfaceanchored and do not self-associate spontaneously. In this scenario, avidity may be explained by the enhanced concentration of ligand binding sites in proximity to the monomeric target, which may favor rebinding of the multiple ligand binding sites with the receptor molecules upon dissociation of the protein complex.
\end{abstract}

Keywords: Kunitz domain, protease inhibitor, proteolysis, serine protease, protein engineering, bivalent interactions, avidity, binding cooperativity.

\title{
Impact statement
}

Lacham-Hartman et al. demonstrate enhancement of binding affinity through avidity in a complex between a bivalent ligand and a soluble monomeric target with a single binding site. Avidity effects have previously been demonstrated only for clustered receptor molecules presenting multiple binding sites. Our model may explain how polyvalent ligands can agonize or antagonize biological interactions involving nonclustered target molecules that are crucial for intra- and extracellular structural, metabolic, signaling, and regulatory pathways. 


\section{Introduction}

Numerous biological activities in nature rely on polyvalent interactions, in which multiple ligands on one entity transiently ${ }^{1}$ or irreversibly ${ }^{2,3}$ bind to multiple targets (e.g., receptors) on another ${ }^{4,5}$. These biological activities include nutrient transport (e.g., hemoglobin), immune recognition (e.g., antibodies), and signal transduction (e.g., receptor tyrosine kinases) $)^{5,6}$. Each non-covalent binding interaction can be characterized by its binding affinity, with the accumulated strength of many individual affinities referred to as functional affinity, or avidity.

Although the majority of polyvalent interactions are collectively much stronger than their corresponding monovalent interactions (i.e., exhibit stronger avidities ${ }^{5}$ ), there are also examples for negative cooperativity in polyvalent binding due to destabilization of one ligand unit by another ${ }^{7}$. In addition, polyvalency can imbue a ligand with novel properties upon binding to a receptor target, such as the ability to cluster ${ }^{8,9}$ or to disengage from surface-anchored receptors ${ }^{10}$. These properties allow polyvalent ligands to either agonize or antagonize biological interactions that are crucial for intraand extracellular structural, metabolic, signaling, and regulatory pathways ${ }^{11}$.

These agonistic and antagonistic effects have been elucidated from systems in which ligands interact with surface-anchored receptors. Examples include transcription factor binding to multiple DNA regions ${ }^{12}$, and influenza virus fusion with the sialic acids found at the host cell surface via the former's hemagglutinin (HA) glycoproteins and the latter's cell adhesion mediation properties ${ }^{13}$. Additional examples include conjugating engineered polypeptides that contain multiple integrin-binding RGD motifs spaced by SGSGSGSG linkers to a surface for cell adhesion ${ }^{14}$, attachment of immune cells to epithelial cells via glycoproteins to generate an immune response ${ }^{15}$, and others (reviewed in ${ }^{5}$ and $^{11}$ ).

Polyvalent interactions may also impact the binding selectivity of a ligand to its target by inducing steric stabilization of specific interactions and/or by enhancing the local concentration of a ligand. The size and distribution of polyvalent ligands in the vicinity of their targets may reduce the interaction of the target with other natural ligands ${ }^{16}$. For example, Lees et al., ${ }^{17}$ have shown that a polymer containing multiple sialic acids was able to inhibit the adhesion of influenza virus to erythrocytes by binding to multiple hemagglutinin (HA) proteins on the virus surface. Moreover, the interaction of this multivalent polymer with multiple HA proteins also prevented binding of specific antibodies to HA due to steric hindrance imposed by the multivalency during binding. Polyvalent ligands may also increase the concentration of ligand binding sites in the proximity of their target receptor, such that when the ligand-receptor protein complex dissociates, the receptor target can re-associate with multiple ligand binding sites such that a polyvalent system favors rebinding with the ligand ${ }^{16,18}$.

In theory these advantages may also confer avidity effects between polyvalent ligands and naturally monomeric, soluble receptors which are incapable of self-association 
except if mediated by the scaffolding of the ligand. Engineering of such polyvalent ligands may provide a novel strategy to enhance both affinity and specificity of proteinprotein interactions. However, the affinity implications of polyvalency in such systems has not been explored, possibly because of a lack of suitable and easily accessible model systems ${ }^{19}$. Therefore, we aimed to close this gap by investigating the effect of avidity on a system in which all the binding sites in the polyvalent protein ligand are fully functional (i.e., no destabilization in the polyvalent ligand) and the target protein, in its soluble form, is monomeric and may not self-associate or cluster. The complex formed between the serine protease mesotrypsin and its cognate inhibitor, the human amyloid precursor protein inhibitor (APPI), from the Kunitz domain family, represents an excellent system for that purpose.

As a result of specific evolutionary mutations, mesotrypsin exhibits a distinctive resistance to almost all biological inhibitors including those of the Kunitz domain family ${ }^{20-24}$. Indeed, compared with other trypsin isoforms, the binding affinities of mesotrypsin with human Kunitz domains are 2-4 orders of magnitude weaker ${ }^{25}$. These low affinities (which are expressed as high inhibition constants $\left(K_{i}\right)$ ) render mesotrypsin a good soluble target for exploration of potential avidity effects, since improvements in the relatively weak binding affinities should be easily detectable.

APPI, which is abundant in nature, is composed of 58-amino acids, with a MW of approximately $6 \mathrm{kDa}^{26}$. The canonical loop within the APPI scaffold serves as a recognition site for mesotrypsin ${ }^{25,27}$, to which it binds as a competitive inhibitor with 1:1 stoichiometric ratio ${ }^{28-30}$ and inhibition constant of about $133 \mathrm{nM}^{28,31}$. Here, we envisioned that the APPI Kunitz domain could be arranged in tandem repeats to generate a polyvalent scaffold, inspired by the natural arrangement of tandem Kunitz domains in bikunin, another Kunitz domain family member. In bikunin, the two nonidentical Kunitz domains occur sequentially in a single protein chain, with each subunit possessing a distinct inhibitory spectrum; the N-terminal domain targets elastases, while the C-terminal domain targets trypsin-like serine proteases ${ }^{32}$.

Here, we generated a stable homodimeric APPI in which both canonical inhibitory loops, one on each monomer, are correctly folded and fully functional. We show that the inhibitory activity of each loop in polyvalent APPI is over three-fold more potent than that of the corresponding loop in monovalent APPI. Using mesotrypsin, which does not self-associate spontaneously, we probed how each inhibitory loop in the dimer could bind each mesotrypsin monomer and thus provide support for mesotrypsin dimerization upon binding to the bivalent APPI. We also propose a simple kinetic model for mesotrypsin - APPI homodimer binding, quantitatively explaining the experimentally observed cooperativity in binding affinity (i.e., avidity). In so doing, we provide a framework for showing that the valency of ligands may affect avidity in protein-protein interactions that include targets that are not surface-anchored and do not self-associate spontaneously. 


\section{Results and Discussion}

Polyvalent interactions play an important role in many biochemical processes ${ }^{5}$. The conjugation of multiple copies of a ligand to a scaffold facilitates the simultaneous interaction of the latter with multiple target molecules, which strengthens binding affinity through an avidity effect ${ }^{16,33,34}$. A key criterion for avidity is prior clustering of the target molecules, such as occurs when receptors are immobilized on a cell surface or solid support antecedent to ligand binding 5,35 . This effect has been found mostly in antibody-antigen interactions ${ }^{1}$ in which the antigen is membrane-anchored. To the best of our knowledge, no attempts have been made to investigate the presence of avidity effects in soluble protein complexes in which a polyvalent ligand interacts with target molecules that are not in close proximity to each other prior to polyvalent protein complex formation. In such instances, it may be less trivial to achieve an avidity effect, as avidity requires that the unanchored, unclustered and freely diffusing monomeric target molecules (which have the potential to interfere with multiple binding to the ligand) exhibit superior binding affinity to polyvalent ligands than to monovalent ligands. The current research was designed to develop and explore avidity under such conditions, that is, when there is no clustering of the target molecules prior to their binding to the polyvalent ligand.

Generating the dimer construct gene in a Pichia pastoris expression system. To examine the feasibility of inducing binding avidity in a soluble protein complex comprised of a polyvalent ligand that binds freely diffusing, unclustered, monomeric target molecules, we chose the APPI-mesotrypsin complex, which was first characterized in Salameh et al. ${ }^{28}$, as proof of concept. To satisfy the first criterion, namely, that each unit of the polyvalent ligand be fully functional, it was necessary to generate a correctly folded recombinant APPI dimer having two fully functional inhibitory units. Next, properties such as ligand density (spacing) and orientation needed to be considered, and therefore special attention was paid to selecting an appropriate linker to achieve optimal conjugation of two APPI monomers to form the dimer. Many studies have suggested that the linker plays a crucial role in facilitating ligand binding and that it can be optimized to allow correct protein folding or to obtain optimal biological activity in fusion proteins ${ }^{14,16,36}$. Accordingly, we used a wellcharacterized flexible linker composed of three repetitions of GlyGlyGlyGlySer (designated GGGGS $\times 3)^{36}$, such that the distance between the APPI inhibitory loops was predicted to allow correct folding of the two monomers and their simultaneous binding to two mesotrypsin units. The gene construct for dimeric APPI was generated by PCR assembly (Fig. S1A) and transformed into P. pastoris. We then extracted the genomic DNA and amplified the target genes with AOX primers. The PCR products were separated by electrophoresis on $1 \%$ agarose gel and the bands representing the 
amplified APPI genes were confirmed, as shown in Fig. S1B. The sequence of the APPI dimer gene construct containing the GGGGS $\times 3$ linker was also verified (Fig. S1C).

Large-scale protein production and purification of APPI variants. The recombinant APPI variants were purified using nickel-affinity chromatography, which is possible because of their C-terminal His tags (Fig. 1A,B). Size exclusion chromatography (SEC) was then used to eliminate impurities, such as imidazole residuals, from the APPI monomer or dimer (Fig. 1C,D). Subsequently, we analyzed the purified proteins on $15 \%$ SDS-PAGE followed by InstantBlue Coomassie staining to confirm the size of the proteins (Fig. 1E,F).

As expected (given a MW of approximately $9 \mathrm{kDa}$ for the APPI monomer containing a His tag and restriction enzyme site ${ }^{26}$ ) dimeric APPI showed a predominant band at MW $15 \mathrm{kDa}$ (Fig. 1F). However, a significant and unexpected difference was observed between APPI dimer concentrations obtained from analytical and functional assays (data not shown). Specifically, whole protein concentrations obtained from the absorbance spectrum of the APPI dimer at $280 \mathrm{~nm}$ were significantly higher than the inhibitory unit concentrations obtained from its catalytic activity in the presence of bovine trypsin. From these observations, we inferred the presence of several recombinant dimeric APPI conformations, of which only some displayed the correctly folded and fully functional canonical loops (i.e., enzyme recognition sites) that serve as inhibitory units in their interaction with trypsins (i.e., bovine trypsin and mesotrypsin). On the basis of the monomeric structure of the APPI Kunitz domain, which contains three intramolecular disulfide bridges, the monomeric units that constitute the APPI dimer may potentially include non-natural, mixed pairs of disulfide bonds that enable only partial protein folding and that are incompatible with correct folding of the inhibitory loop. During protein folding, the correct formation of intramolecular disulfide bonds within each monomeric unit of the APPI dimer is essential for the activity of the inhibitory loop ${ }^{37}$. It is particularly crucial in the context of enzymeinhibitor complexes, such as trypsin-APPI, in which the inhibitory loop of APPI binds to the active site of mesotrypsin in a site specific manner, as per the lock-and-key interaction model ${ }^{38}$. 

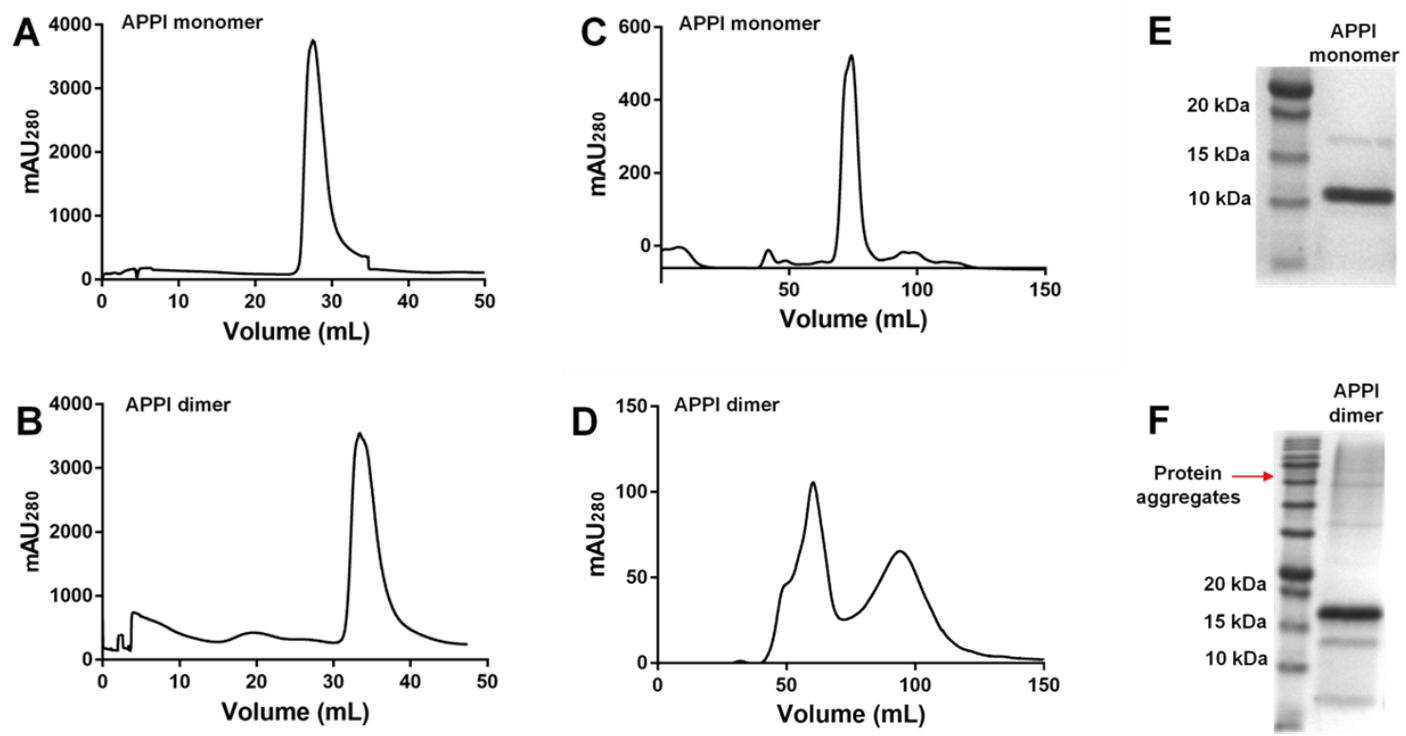

Figure 1: Purification of APPI variants. Nickel affinity chromatography (A and B) and size-exclusion chromatography (SEC) (C and D) of recombinant amyloid precursor protein inhibitor (APPI) monomers (A and $\mathbf{C})$ and dimers (B and $\mathbf{D})$. The left peak in (D) is dimeric APPI, whereas the right peak is imidazole, which was used for protein elution in the nickel column. For all chromatograms, the $\mathrm{X}$-axis represents the elution volume and the $\mathrm{Y}$-axis represents absorbance at $280 \mathrm{~nm}$. 15\% SDS-PAGE analysis of the SEC-purified APPI variants $(\mathbf{E}$ and $\mathbf{F})$. 
To generate an APPI dimer in which both the displayed canonical inhibitory loops were fully functional, we applied an additional protein purification step involving affinity chromatography with a bovine trypsin column (Fig. 2A) to isolate the fraction of dimeric APPI that is correctly (and fully) folded. Two separate peaks were observed and analyzed by 15\% SDS-PAGE (Fig. 2B). The protein fraction represented by Peak 2 exhibited superior inhibition of mesotrypsin than the protein fraction represented by Peak 1 (data not shown). We then compared the dimeric APPI fractions corresponding to affinity chromatography Peaks 1 and 2 with monomeric APPI by means of an additional 15\% SDS-PAGE analysis (Fig. 2C), and these fractions were then used for further functional characterization of the APPI protein dimer. Next, we determined the monomeric and dimeric APPI protein concentrations using absorbance at $280 \mathrm{~nm}$ (with extinction coefficients of 13,325 and $22,180 \mathrm{M}^{-1} \mathrm{~cm}^{-1}$, respectively). Approximately $20 \mathrm{mg}$ and $0.4 \mathrm{mg}$ of purified APPI monomer and dimer were produced from a $1 \mathrm{~L}$ yeast culture, respectively. Finally, MALDI-TOF spectra confirmed the MW of the purified monomeric and dimeric APPI variants (Fig. S2A,B). 
A

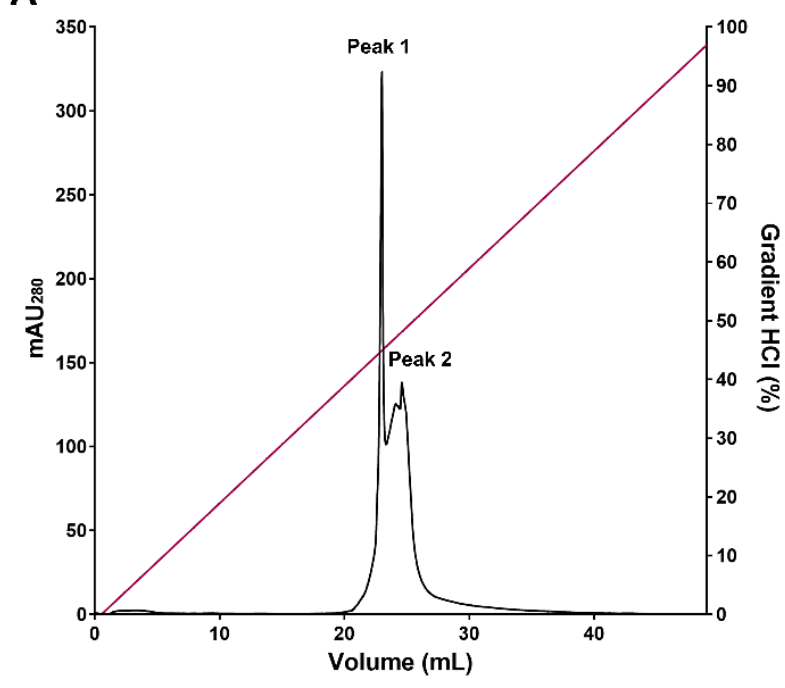

B

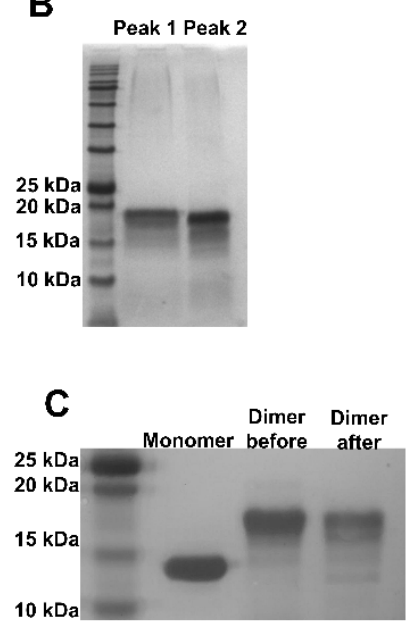

Figure 2: Final purification step to obtain fully functional dimeric APPI. Using an affinity chromatography approach, dimeric APPI was eluted from a bovine trypsin column using a $0-100 \mathrm{mM} \mathrm{HCl}$ gradient (A). The protein fraction eluted in Peak 2 was chosen for further functional (protease inhibition) experiments, because it exhibited greater inhibition potency toward trypsin in comparison with the protein fraction eluted in Peak 1 (data not shown). 15\% SDS-PAGE analysis of the purified dimeric APPI fractions (B) and comparison of purified monomeric APPI with dimeric APPI before and after purification $(\mathbf{C})$. 


\begin{abstract}
APPI dimer titration analysis is consistent with 1:2 APPI:mesotrypsin binding stoichiometry. To determine whether APPI dimer can simultaneously engage two molecules of trypsin, the APPI inhibitory unit concentration (in the APPI monomer and dimer) was determined by trypsin titration analysis and compared with concentrations determined by absorbance (Table S1). To determine concentrations by titration assay (Fig. 3), we used a known quantity of bovine trypsin, which has known molecular mass, and our two inhibitors (APPI monomer and dimer), which have known molecular masses. A comparison between the concentrations of active inhibitory units determined from the titration (Fig. 3) with the values that would be expected for 1:2 APPI:trypsin binding stoichiometry was highly consistent, whereas nearly double the amount of trypsin was quenched as could be explained by a 1:1 binding model. These data provide evidence that APPI dimer can bind simultaneously to two molecules of trypsin.
\end{abstract}


A

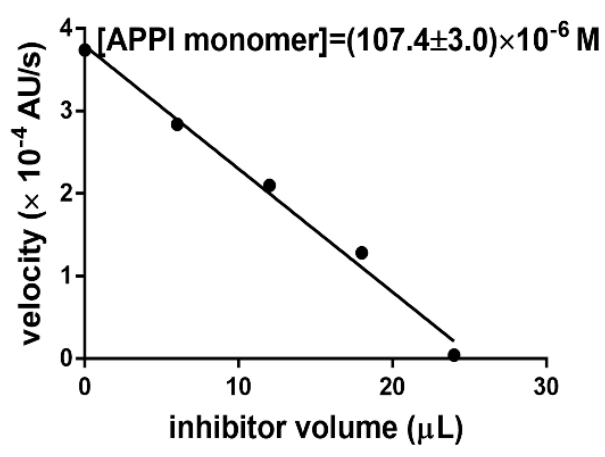

B

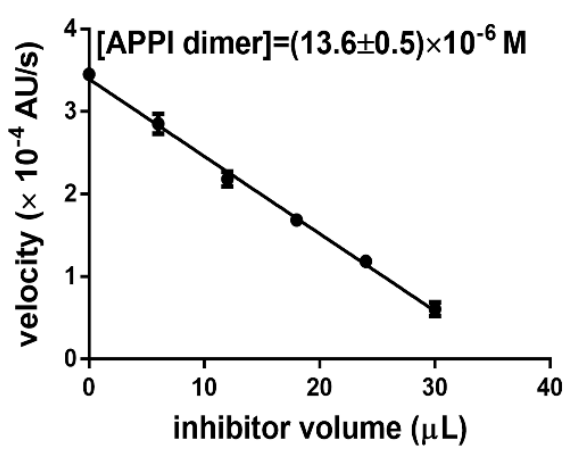

Figure 3: Titration of inhibition units. Bovine trypsin titration curves of monomeric (A) and dimeric (B) APPI. The X-axis represents the APPI volumes added to the reaction and the $\mathrm{Y}$-axis represents the reaction velocity (i.e., velocity of substrate cleavage by bovine trypsin) at several APPI dilutions. The experiment setup is described in the Materials and Methods section. 
The mesotrypsin affinity of a single inhibitory unit is enhanced in dimeric compared with monomeric APPI. To assess the binding of monomeric and dimeric APPI to mesotrypsin, we carried out enzyme inhibition experiments that monitored cleavage of the chromogenic substrate Z-GPR-pNA by mesotrypsin in the presence of varying APPI concentrations. First, we determined the Michaelis-Menten constant $\left(K_{m}\right)$ of z-GPR-pNA binding to mesotrypsin. As shown in Fig. 4A, the $K_{m}$ constant was calculated using nonlinear regression fitting to the Michaelis-Menten equation. The obtained value was $25.1 \pm 2.4 \mu \mathrm{M}$, consistently with previous studies ${ }^{31}$. Next, we determined the affinity of the inhibitory units in monomeric and dimeric APPI proteins for mesotrypsin using a competitive inhibition model to calculate the inhibition constant 28,31 . We observed a classic competition pattern of inhibition for both inhibitors (Fig. 4B, C). We found that the inhibition constant of a single inhibitory unit in the APPI dimer was $K_{i}=36.1 \pm 2.2 \mathrm{nM}$ (Fig. 4C), which indicates an almost 3.4-fold enhancement of affinity compared with the inhibition constant of a single inhibitory unit in the APPI monomer $\left(K_{i}=122.5 \pm 10.0 \mathrm{nM}\right)$ (Fig. 4B), whose $K_{i}$ value was consistent with previous studies ${ }^{28,31}$. This enhancement in affinity may result from the mesotrypsin protein dimerizing upon binding the APPI dimer and the simultaneous binding of the two mesotrypsin active sites (in the mesotrypsin dimer) to the two APPI canonical binding loops (in the APPI dimer), as discussed in the next section. 

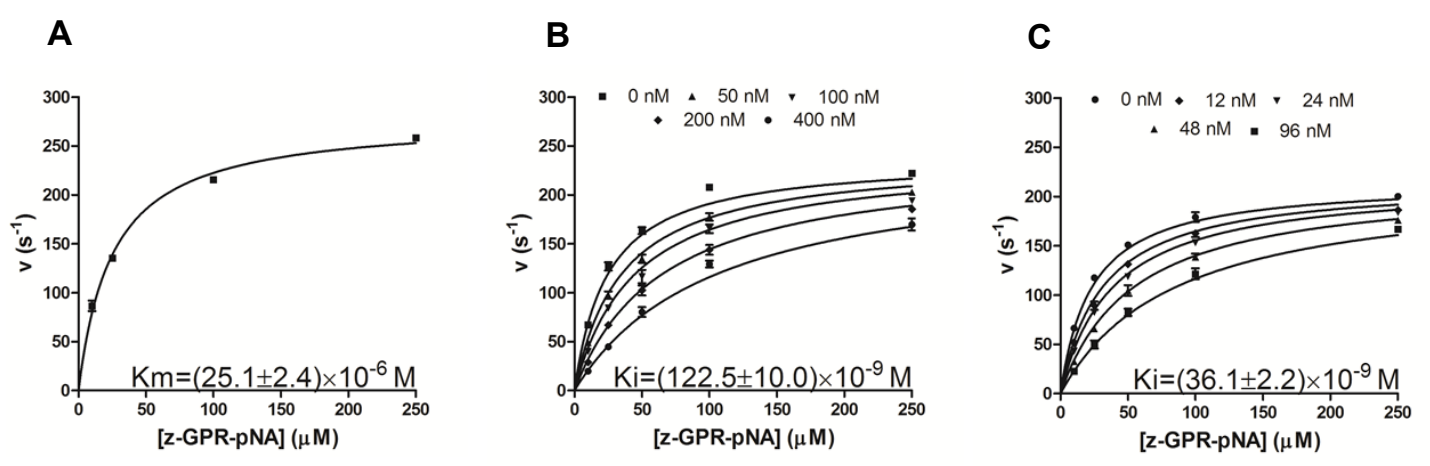

Figure 4: Determination of inhibition constants. Determination of the MichaelisMenten constant $\left(\mathrm{K}_{\mathrm{m}}\right)$ for binding of the chromogenic substrate Z-GPR-pNA to mesotrypsin (A). Kinetics of mesotrypsin inhibition by the APPI monomer (B) and dimer (C). In all experiments, substrate concentration was 5-250 $\mu \mathrm{M}$ and enzyme concentration was $0.25 \mathrm{nM}$. The concentration of the canonical APPI inhibition unit was $0-400 \mathrm{nM}$ for the monomer and $0-96 \mathrm{nM}$ for the dimer. Reactions were followed spectroscopically for $5 \mathrm{~min}$, and initial rates were determined from the increase in absorbance caused by the release of p-nitroaniline (at $410 \mathrm{~nm}$ ). Data were globally fitted by multiple regression to the classic competitive inhibition equation (Equation 1; see Materials and Methods). 
Kinetic model for APPI homodimer binding to mesotrypsin. In order to quantitatively understand the observed cooperativity in binding affinity (i.e., avidity) for APPI homodimer - mesotrypsin complex, we propose a simple kinetic model that goes beyond the standard Michaelis-Menten model for competitive enzyme inhibition (Fig. 5). The proposed model takes into account the important fact that a large fraction of APPI homodimer binds two mesotrypsin molecules simultaneously. This dual binding enhances the propensity for mesotrypsin dimerization via reversible binding to APPI homodimers which in turn leads to the cooperativity in APPI homodimer mesotrypsin binding affinity (i.e., avidity). This mechanism is similar to the previously proposed model for DNA-linked nanoparticles ${ }^{39,40}$. In particular, we assume that the following kinetic scheme describes the inhibition of mesotrypsin by APPI homodimers (Fig. 5).

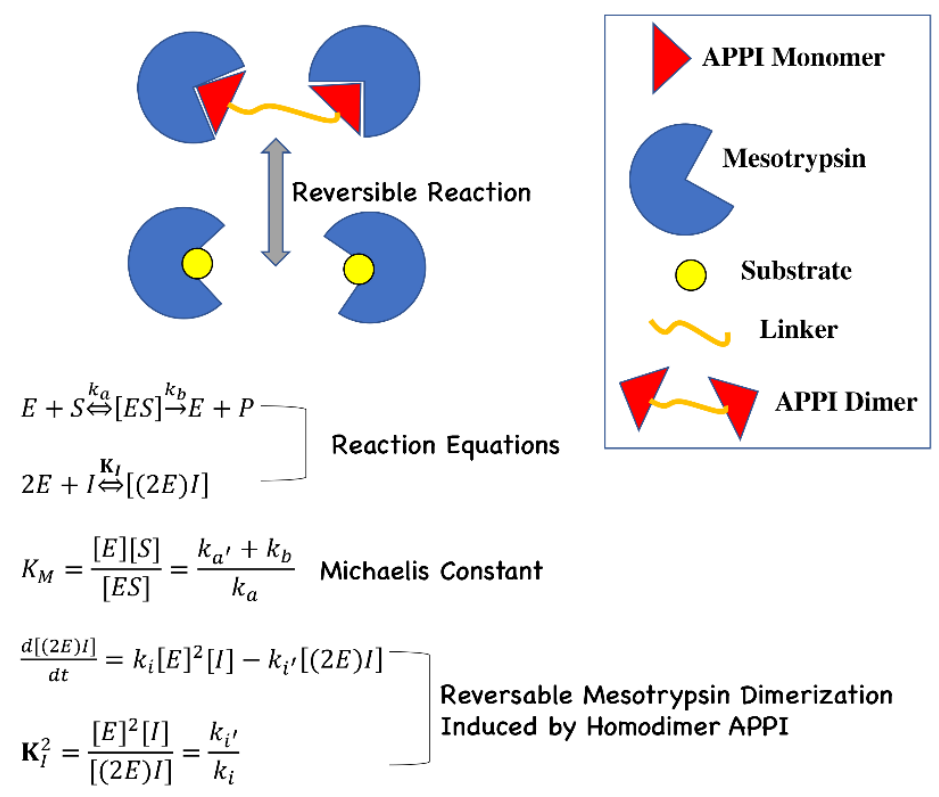

Figure 5: Proposed reaction mechanism for the APPI homodimer induced mesotrypsin dimerization. The presence of transiently bound APPI homodimers modifies the binding reaction order from being linear in the enzyme concentration (i.e., the classical Michaelis-Menten competitive enzyme inhibition) to $k_{i}[E]^{2}[I]$, inducing mesotrypsin dimerization. Here $[E]$ is the mesotrypsin concentration, $[S]$ is the substrate concentration, $[I]$ is the APPI homodimer concentration, $k_{i}$ is the association rate constant of the APPI homodimer - mesotrypsin binding reaction, and $k_{i}$, is the corresponding dissociation rate constant; $k_{a}$ is the associate rate constant of the mesotrypsin - substrate binding reaction, and $k_{a}$, is the corresponding dissociation rate constant; $k_{b}=k_{\text {cat }}$ is the catalytic constant; $\mathbf{K}_{I}$ is the equilibrium inhibition constant, and $K_{M}$ is the Michaelis constant, and $[P]$ stands for the enzymatic reaction product concentration. 


$$
\begin{aligned}
& E+S \stackrel{k_{a}}{\Leftrightarrow}[E S] \stackrel{k_{b}}{\rightarrow} E+P \\
& 2 E+I \stackrel{\mathbf{K}_{I}}{\Leftrightarrow}[(2 E) I]
\end{aligned}
$$

and the corresponding kinetic equation for Eq. 2:

$$
\frac{d[(2 E) I]}{d t}=k_{i}[E]^{2}[I]-k_{i^{\prime}}[(2 E) I]
$$

Therefore, in equilibrium we define the following inhibition constant, $\mathbf{K}_{I}$, for the homodimer APPI inhibitor:

$$
\mathbf{K}_{I}^{2}=\frac{[E]^{2}[I]}{[(2 E) I]}=\frac{k_{i^{\prime}}}{k_{i}}
$$

We stress the important fact that unlike the conventional Michaelis-Menten scheme for competitive enzyme inhibition, here the enzyme concentration enters via a non-linear term, i.e., quadratic in the mesotrypsin concentration, $k_{i}[E]^{2}[I]$ (Fig. 5). In equilibrium, Eq. 3 leads to the following quadratic equation:

$$
[E S]^{2} \frac{2[I]}{\mathbf{K}_{I}^{2}}\left(\frac{K_{M}}{[S]}\right)^{2}+[E S]\left(1+\frac{K_{M}}{[S]}\right)-E_{t o t}=0
$$

Where $K_{\mathrm{M}}=\left(k_{a},+k_{b}\right) / k_{a}$ is the Michaelis constant. The solution of this equation:

$$
[E S]=\frac{-\left(1+\frac{K_{M}}{[S]}\right)+\sqrt{\left(1+\frac{K_{M}}{[S]}\right)^{2}+8 E_{t o t}\left(\frac{K_{M}}{[S]}\right)^{2} \frac{[I]}{\mathbf{K}_{I}^{2}}}}{4\left(\frac{K_{M}}{[S]}\right)^{2} \frac{[I]}{\mathbf{K}_{I}^{2}}}
$$

and the resulting reaction velocity:

$$
v=k_{b}[E S]
$$

Fitting Eq. 7, to the experimental data, allows us to obtain the value of $\mathbf{K}_{I}$ (Fig. 6). The fits to kinetic measurements performed at different APPI homodimer concentrations show an excellent agreement with the data and provide the average value for the equilibrium inhibition constant, $\mathbf{K}_{I} \approx 1.8 \mathrm{nM}$. We stress the fact that this equilibrium inhibition constant, $\mathbf{K}_{I}$, cannot be directly compared to the corresponding equilibrium constant extracted above (using the conventional Michaelis-Menten model), since $\mathbf{K}_{I}$ is obtained using a different kinetic mechanism, Eq. 3. Intuitively, the observed cooperativity in binding affinity (i.e., avidity) of APPI homodimer, as compared to APPI monomer, stems from the fact that the APPI homodimer - mesotrypsin association rate, $k_{i}[E]^{2}[I]$, scales as a second power of the enzyme concentration, leading to a more efficient inhibition. This is unlike the APPI monomer case, where the APPI - mesotrypsin association rate, $\kappa_{i}[E][I]$, scales linearly in the enzyme concentration; here $\kappa_{i}$ is the association rate constant of the mesotrypsin - monomer APPI binding reaction. 

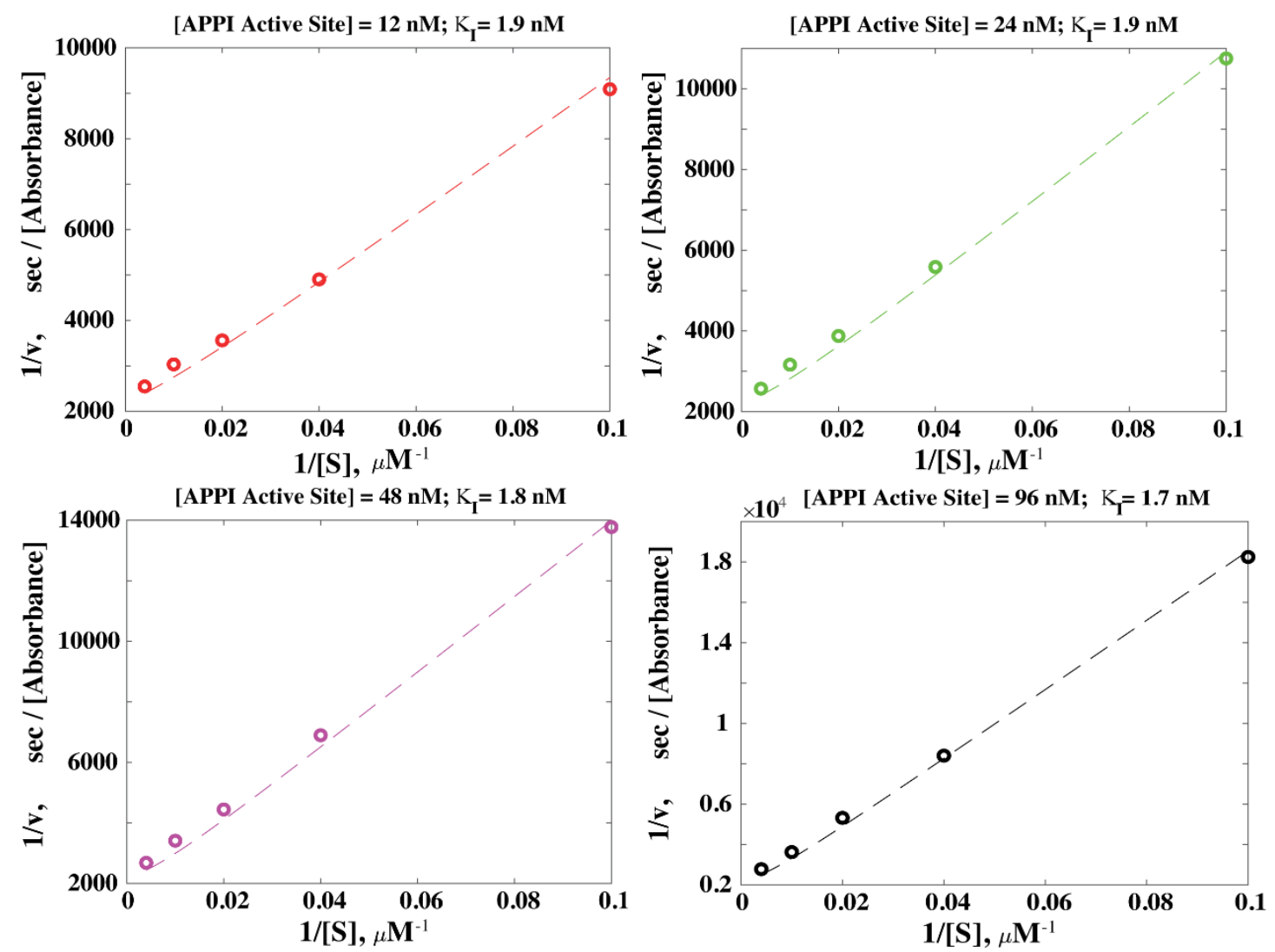

Figure 6. Non-linear model for mesotrypsin inhibition by APPI homodimers provides an excellent fit to experimental data. Fitting the reaction velocity (from Fig. 4) measured at different substrate concentration (using Eqs. 6 and 7), allows us to extract the equilibrium inhibition constant $\mathbf{K}_{I}$. Each of the four plots shows the fit corresponding the measurements performed at a given APPI homodimer concentration $[I$, where [APPI Active Site] $=2[I]$, stands for the concentration of APPI monomer units. The added mesotrypsin concentration was maintained at $\left[E_{t o t}\right]=0.25 \mathrm{nM}$. The fitted values of $\mathbf{K}_{I}$ are presented above each plot.

The increase in affinity measured here for a dimeric compared with a monomeric inhibitor-enzyme complex is much smaller than that known for polyvalent compared with monovalent antibody-antigen complexes. In our study, avidity occurs in the context of the interaction between a bivalent inhibitor and a monomeric enzyme with a single active site, where the enzyme is in solution and unclustered prior to binding to the bivalent inhibitor. By contrast, in antibody-antigen complexes, the antigen (or receptor) molecules are anchored to a surface (e.g., to a cell membrane) and thus are clustered in close proximity to each other before they bind the multivalent antibody ligand. Such clustering significantly enhances interaction strength, such that affinity in polyvalent antibody-antigen complexes is $2-3$ orders of magnitude stronger than in their monovalent analogues ${ }^{41,42}$. When monomeric target molecules with a single 
binding site are in solution, the molecules are separated from each other and have greater mobility than clustered (or surface anchored) target molecules and binding sites. This may make the binding of the monomeric targets to a dimeric ligand more difficult if they follow a 'lock and key' mode of molecular recognition. In contrast, during interactions with antibodies, the organization of surface-immobilized targets exhibits a high surface epitope density that allows them to rebind the multivalent ligand much faster and in the correct conformation for optimal binding. This point was recently demonstrated by Hadzhieva et al. ${ }^{35}$ in a study that elucidated the correlation between the binding affinities of several IgG antibodies and the density of an antigen attached to a surface. The authors chose several antibodies that bound the HIV envelope glycoprotein 120 (namely gp120) with a wide range of affinities. Using a surface plasmon resonance (SPR) spectroscopy method to evaluate affinity, they controlled the density of antigen immobilized to the surface of a sensor chip and performed avidity measurements. As antigen density increased, enhanced avidity was observed for all the antibodies, as expressed by a systematic decrease in their equilibrium dissociation constants driven largely by reductions in their dissociation rate constants. For example, for the HJ16 antibody, a 60-fold increase in gp120 density led to a 150-fold decrease in the dissociation rate constant that was expressed as a 168 -fold increase in binding affinity. One of the proposed explanations for this result was that the high density of gp120 allowed the formation of an IgG hexametric structure, which resulted in enhanced avidity ${ }^{35}$. Taken together, it is plausible that a greater avidity effect is observed in systems that confer both high target density and restricted target movement (as in surface-immobilized antigens or membrane-anchored receptors). This assumption is supported by the fact that proper orientation of free target molecules is a prerequisite for optimal ligand binding. The formation of a soluble complex, such as in interactions between an inhibitor and its target enzyme, requires the specific orientation of the interface residues of both interacting proteins. Obtaining the optimal orientation for binding is much more difficult for soluble complexes because of the dynamic and constant movement of the target molecules in solution, whereas immobilized targets with restricted movements may confer faster association rates and higher affinity in equilibrium.

In other words, the binding epitopes of a surface-anchored protein target may be subject to conformational constraints that may result in lower entropic penalties upon binding to a ligand, which enhances binding affinity ${ }^{5,43}$. For monomeric protein targets in solution, the structure of the target is flexible, such that there is a large difference in the structural rigidity of the protein target between its unbound state and its state when bound to its polyvalent ligand. In contrast, the structure of the target is more rigid when immobilized to a surface, such that there is little difference in rigidity between its unbound and bound states. This conformational constraint may also be achieved by target dimerization, as observed for mesotrypsin in our system. 
The positive cooperativity we observed between APPI units binding to mesotrypsin stands in contrast to the results obtained by Farooq's group, who characterized ligand binding to the two WW tandem domains of the YAP2 protein, a transcriptional factor that regulates genes related to cell fate ${ }^{7}$. They isolated the WW domains and determined the binding affinity of each WW domain toward each ligand from equilibrium dissociation constants using isothermal titration calorimetry measurements. Next, they compared the constants obtained for a specific ligand with the binding affinity of the same ligand toward the native structure (i.e., to YAP2 containing two WW domains). They found negative cooperativity during ligand binding to tandem WW domains. Specifically, each ligand exhibited higher affinity for the isolated WW domain in comparison with the tandem, fused WW domains of the native YAP2 protein. The researchers explained this negative cooperativity by positing that inter-domain interactions arising from the proximity of the two WW domains to each other impede or sterically hinder ligand binding to each domain.

Bikunin (like APPI) is a member of the Kunitz family, however (unlike APPI) it has two subunits that are linked via a single peptide bond. Similarly to the WW domain in YAP2, bikunin cannot bind two serine protease simultaneously. Ollis's group postulated that the native structure of bikunin can control the proteolytic activity of enzymes because each domain binds a different protease and so acts as a useful regulatory mechanism, with one Kunitz domain diminishing or abolishing binding at the second domain ${ }^{32}$.

These previous studies and our own current study include examples of soluble systems that have two domains within a single protein ligand, with each domain having a single binding site capable of interacting with monomeric protein targets in solution. However, the major difference between our study and the previous studies of bikunin and the WW domains in YAP2 is that their tandem domains, when embedded in the entire protein, do not exhibit enhanced binding affinity, with the lack of enhancement attributed to steric interference. On the other hand, in our system the affinity of the target molecule mesotrypsin to a single binding loop in dimeric APPI was enhanced relative to the binding of mesotrypsin to the same binding loop in monomeric APPI. This was probably because there was negligible steric interference between the single APPI units (and specifically the canonical inhibitory loops) within the dimeric APPI structure. In the absence of steric hindrance, our dimeric APPI could simultaneously bind, via its canonical binding loops, two mesotrypsin units as shown in our APPI dimer titration analysis. In addition, our dimeric APPI protein is a homodimer, whereas the WW domains of the YAP-2 protein and the two Kunitz domains of bikunin differ in their amino acid content (i.e., they are heterodimers) which may also influence their target binding kinetics. 


\section{Conclusions}

In summary, we have demonstrated the feasibility of enhancing binding affinity through an avidity effect using a complex between mesotrypsin and bivalent APPI, in which the mesotrypsin target protein is monomeric in solution (prior to binding) and has a single binding site. Isolation of the fully active recombinant dimeric APPI exhibiting correct folding and possessing two fully functional inhibitory loops (one on each monomer) allowed us to achieve accurate results, unaffected by unfolded or partially unfolded protein domains. Binding affinity, which was measured for a single inhibitory loop, was 3.4-fold stronger for dimeric APPI compared with monomeric APPI. We revised a conventional Michaelis-Menten kinetic model for competitive inhibition, introducing a non-linear effect induced by the presence of bivalent APPI homodimer. This model provides a quantitative explanation for the observed enhanced tendency for mesotrypsin dimerization leading to cooperativity (i.e., avidity) in the binding affinity. Moreover, we validated the ability of dimeric APPI to bind two mesotrypsin units by using binding titration analysis, indicating that the enzyme recognition site on each of the two inhibitory units possesses a canonical loop that is available for simultaneous interaction with trypsins.

\section{Materials and methods}

Reagents. Synthetic oligonucleotides were obtained from Integrated DNA Technologies (Coralville, IA, USA). Restriction enzymes, T4 ligase, and Q5 polymerase were purchased from New England Biolabs (Ipswich, MA, USA), and nucleoside triphosphates (dNTPs) from Jena Bioscience (Jena, Germany). The methylotrophic yeast $P$. pastoris strain GS115 and the Pichia expression vector (pPIC9K) were obtained from Invitrogen (Carlsbad, CA, USA). Bovine trypsin, disuccinimidyl suberate (DSS), and the chromogenic substrates benzyloxycarbonylGly-Pro-Arg-p-nitroanilide (Z-GPR-pNA), 4-nitrophenyl 4-guanidinobenzoate (pNPGB), and benzoyl-L-arginine-p-nitroanilide (L-BAPA) were purchased from Sigma-Aldrich (St. Louis, MO, USA). Rabbit anti-trypsin antibody (Ab-200997) and mouse anti-his tag (Ab-49936) were obtained from Abcam (Cambridge, UK). Affi-gel 10 resin was obtained from Bio-Rad laboratories (Hercules, CA, USA).

Generation of APPI gene constructs in expression vector pPic9k. APPI monomer (PDB:3L33, residues 4-55) was cloned into the plasmid pPic9k as previously described 31 and the gene construct encoding dimeric APPI was generated by a gene assembly method. Briefly, pPic9k plasmid containing the full-length APPI gene was amplified by polymerase chain reaction (PCR) using Q5 DNA polymerase with primers containing appropriate restriction sites for $\mathrm{pPic} 9 \mathrm{k}$ plasmid and the addition of GGGGS $\times 3$ linker at the C-terminal of APPI unit. The following primers were used to 
amplify the monomeric APPI: APPI FW: (5'TGCTACGTATTAATTAACGAAGTTTGTTCTGAACAAGCTG-3') and APPI RC: $\left(5^{\prime}-\right.$

GCAATGGAATTCGGATCCCCCTCCTCCGGATCCTCCCCCTCCGGAACCTCC CCCTCCAATAGCAGAACCACAAACAGC-3').

Both the amplified PCR fragments and pPick9k plasmid were digested with the SnaBI and EcoRI restriction enzymes to generate an APPI dimer gene construct (Fig. S1A). The digested fragments were ligated using T4 ligase, transformed into E. coli, and plated on plates with lysogeny broth containing ampicillin (LB-amp). Finally, the dimeric APPI gene construct was sequenced at the DNA Microarray and Sequencing Unit of the National Institute for Biotechnology in the Negev at Ben Gurion University (DMSU, NIBN, BGU). Expression vectors were linearized by SacI digestion and used to transform $P$. pastoris strain GS115 by electroporation. This resulted in the insertion of the construct at the first alcohol oxidase (AOX1) locus of P. pastoris, thereby generating a His + Mut + phenotype. Transformants were selected for the His + phenotype on $2 \%$ agar containing regeneration dextrose biotin (RDB; $18.6 \%$ sorbitol, $2 \%$ dextrose, $1.34 \%$ yeast nitrogen base, $4 \times 10^{-5} \%$ biotin, and $0.005 \%$ each of Lglutamic acid, L-methionine, L-lysine, L-leucine, and L-isoleucine) and allowed to grow for 3 days at $30^{\circ} \mathrm{C}$. Cells were harvested from the plates and subjected to further selection for high copy number on the basis of their growth on $2 \%$ agar containing $1 \%$ yeast extract, $2 \%$ peptone, $2 \%$ dextrose medium, and the antibiotic G418 (Geneticin, 4 $\mathrm{mg} / \mathrm{mL}$, Invitrogen). To verify direct insertion of the construct at the AOX1 locus of $P$. pastoris, the genomic DNA of the highest APPI-expressing colony from each APPI variant was extracted and amplified by PCR with an AOX1 upstream primer, 5'GACTGGTTCCAATTGACAAGC-3', and an AOX1 downstream primer, 5'GCAAATGGCATTCTGACATCC-3' (Fig. S1B). The PCR products were separated on $1 \%$ agarose gel, purified, and the correct sequence of the APPI dimer was confirmed by DNA sequencing analysis (DMSU, NIBN, BGU) (Fig. S1C).

Large-scale purification of APPI variants. P. pastoris cultures expressing either monomeric or dimeric APPI were grown in $50 \mathrm{~mL}$ of BMGY medium $(1 \%$ yeast extract, $2 \%$ peptone, $0.23 \% \mathrm{KH}_{2} \mathrm{PO}_{4}, 1.18 \% \mathrm{~K}_{2} \mathrm{HPO}_{4}, 1.34 \%$ yeast nitrogen base, $4 \times 10^{-}$ $5 \%$ biotin, and $1 \%$ glycerol) overnight and then in $0.5 \mathrm{~L}$ of BMMY medium (similar to BMGY, but with $0.5 \%$ methanol instead of $1 \%$ glycerol) for 3 days, with $2 \%$ methanol being added every $24 \mathrm{~h}$ to maintain induction. Following 4 days of induction, the culture was centrifuged at $3800 \times \mathrm{g}$ for $10 \mathrm{~min}$ and the supernatant containing the secreted recombinant inhibitors was prepared for purification by nickel-immobilized metal affinity chromatography (IMAC). The supernatant was adjusted to $10 \mathrm{mM}$ imidazole and $0.5 \mathrm{M} \mathrm{NaCl}$ at $\mathrm{pH} 8.0$ and left to stand for $1 \mathrm{~h}$ at $4{ }^{\circ} \mathrm{C}$. Thereafter, filtration was performed to remove any additional precipitation using a $0.22 \mu \mathrm{m}$ Steritop bottle-top 
filter (Millipore, MA, USA). The filtered supernatant was loaded on a HisTrap $5 \mathrm{~mL}$ column (GE Healthcare, Piscataway, NJ) at a flow rate of $0.5 \mathrm{~mL} / \mathrm{min}$ for $24 \mathrm{~h}$, washed with a washing buffer (20 mM NaK, $0.5 \mathrm{M} \mathrm{NaCl}$, and $10 \mathrm{mM}$ imidazole (pH 8.0)), and eluted with an elution buffer (20 mM NaK, $0.5 \mathrm{M} \mathrm{NaCl}$, and $0.5 \mathrm{M}$ imidazole) in an ÄKTA-Pure instrument (GE Healthcare, Piscataway, NJ). The buffer of the eluted proteins was replaced with a mesotrypsin buffer $(100 \mathrm{mM}$ tris(hydroxymethyl)aminomethane (Tris), $1 \mathrm{mM} \mathrm{CaCl}_{2}$ (pH 8.0)) using a $3.5 \mathrm{kDa}$ cutoff dialysis kit (Gene Bio Application, Israel). For all recombinant proteins (both monomeric and dimeric APPI), gel filtration chromatography was performed using a Superdex 75 16/600 column (GE Healthcare, Piscataway, NJ) equilibrated with a mesotrypsin buffer $\left(100 \mathrm{mM}\right.$ Tris, $\left.1 \mathrm{mM} \mathrm{CaCl}_{2}(\mathrm{pH} 8.0)\right)$ at a flow rate of $1 \mathrm{~mL} / \mathrm{min}$ on an ÄKTA-Pure instrument (GE Healthcare, Piscataway, NJ). The purified proteins were analyzed by sodium dodecyl sulphate-polyacrylamide gel electrophoresis (SDSPAGE) on a $15 \%$ polyacrylamide gel under reducing conditions followed by staining with InstantBlue Coomassie Protein Stain (Expedeon, Cambridge, UK). Finally, the correct mass of the proteins was validated using a Matrix-Assisted Laser Desorption/Ionization-Time of Flight (MALDI-TOF) REFLEX-IV (Bruker) mass spectrometer (Ilse Katz Institute for Nanoscale Science \& Technology, BGU; Fig.S2).

Preparation of the trypsin column. Affi-gel 10 beads $(8 \mathrm{~mL})$ were mixed with isopropanol, transferred into a $15 \mathrm{~mL}$ falcon tube, and centrifuged at $1000 \mathrm{rpm}$ for 10 min. Then, the isopropanol was discarded, and the beads were washed with cold doubledistilled water (DDW; $10 \mathrm{~mL}$ ) and centrifuged at $2000 \mathrm{rpm}$ for $10 \mathrm{~min}$. This procedure was repeated three times to remove the isopropanol residues for efficient coupling of bovine trypsin to the beads. Thereafter, bovine trypsin powder $(29.5 \mathrm{mg})$ was dissolved in $100 \mathrm{mM}$ HEPES pH $7.5(3 \mathrm{~mL})$ and added to the falcon tube that contained the resin. The mixture was left rotating overnight at $4{ }^{\circ} \mathrm{C}$. The conjugated beads were then packed into a $9 \mathrm{~mL}$ glass column (Bio-Rad laboratories, Hercules, CA, USA). The dimeric APPI $(15 \mathrm{~mL})$ was loaded onto the column and then eluted with a gradient of $0-100 \%$ using $100 \mathrm{mM} \mathrm{HCl}$.

Enzymes and substrates. Recombinant human mesotrypsinogen was expressed in $E$. coli, extracted from inclusion bodies, refolded, purified, and activated with bovine enteropeptidase, as described previously ${ }^{44,45}$. The concentrations of mesotrypsin and bovine trypsin were determined by active site titration using p-nitrophenyl 4guanidinobenzoate hydrochloride (pNPGB) substrate (Sigma-Aldrich, St. Louis, MO, USA), which served as an irreversible inhibitor ${ }^{46}$. For determination of substrate concentration, an aliquot of the chromogenic substrate $\mathrm{N}$ - $\alpha$-benzoyloxycarbonylglycylprolylarginine p-nitroanilide (Z-GPR-pNA) dissolved in dimethyl sulfoxide (DMSO) was incubated with an excess of bovine trypsin powder to obtain full substrate 
cleavage. The substrate concentration was determined from change in absorbance at $410 \mathrm{~nm}$ as a result of the release of p-nitroaniline $\left(\varepsilon_{410}=8480 \mathrm{M}^{-1} \mathrm{~cm}^{-1}\right)$.

Determination of the inhibitory unit concentration by trypsin titration. In the canonical APPI monomer, the enzyme recognition site is a single loop, whereas in a fully functional APPI homodimer, enzyme binding should involve two loops. Since APPI inhibits the activity of serine protease mesotrypsin, each APPI loop or binding site may be referred to as an inhibitory unit. To accurately measure the concentration of inhibitory units available to interact with the protease in the APPI monomer and dimer, titration was performed with pre-titrated bovine trypsin and Na-benzoyl-Larginine 4-nitroanilide hydrochloride (L-BAPA; Sigma-Aldrich, St. Louis, MO, USA), as described previously ${ }^{44}$. Briefly, an assay cocktail $\left(312 \mu \mathrm{l} ; 100 \mathrm{Mm}\right.$ Tris, $5 \mathrm{mM} \mathrm{CaCl}_{2}$ $(\mathrm{pH}=8.0)$ ) having a final concentration of $100 \mu \mathrm{M}$ L-BAPA was added into a 96-well microplate (Greiner, Kremsmünster, Austria). Bovine trypsin enzyme ( $9 \mu \mathrm{L}, 30 \mu \mathrm{M})$ was mixed with each of six dilutions of APPI $(0-6 \mu \mathrm{M}$, prepared from a $9 \mu \mathrm{M}$ inhibitor stock solution) to produce six enzyme-inhibitor mixtures (final volume $45 \mu \mathrm{L}$ ). An aliquot $(8 \mu \mathrm{L})$ of each enzyme-inhibitor mixture was transferred into the wells of a $96-$ well microplate to initiate the reaction. Reactions were monitored spectroscopically at $410 \mathrm{~nm}$ for $5 \mathrm{~min}$ at $37^{\circ} \mathrm{C}$. The reaction velocity (being the change in absorbance with time during substrate cleavage) was calculated for each inhibitor concentration, and the values were plotted versus the volume of inhibitor (0-30 $\mu \mathrm{L}$ APPI) in the enzymeinhibitor mixture. From these data, the X-intercept was determined, and the inhibitory unit concentrations were calculated using equation 8. Reported inhibitory unit concentrations are the average values obtained from two independent experiments, reported as mean $\pm \mathrm{SD}$.

$$
[I U]=D F_{i} V_{e} \frac{[E]}{X_{i n t}}
$$

Where $[\mathrm{IU}]$ is the inhibitory unit concentration $(\mu \mathrm{M})$ of the APPI monomer or dimer, $\mathrm{DF}_{\mathrm{i}}$ is the inhibitor dilution factor (11.5 and 1.7 dilution from the monomeric and dimeric APPI stock solution, respectively), $\mathrm{V}_{\mathrm{e}}$ is enzyme volume (i.e., $9 \mu \mathrm{L}$ bovine trypsin), [E] is enzyme concentration (i.e., $30 \mu \mathrm{M}$ ), and $X_{\text {int }}$ is the value of the $\mathrm{x}$ intercept on a plot of reaction velocity (AU/s) versus inhibitor (APPI) volume $(\mu \mathrm{L})$.

Inhibition Studies. The inhibition constants $\left(K_{i}\right)$ of monomeric and dimeric APPI in complex with mesotrypsin were determined according to the previously described methodology, with minor changes ${ }^{44}$. Briefly, stock solutions of enzyme, substrate, and APPI monomer (or dimer) were prepared at $40 \times$ the desired final concentrations. Assays were performed at $37{ }^{\circ} \mathrm{C}$ in the presence of different concentrations of Z-GPR- 
pNA substrate $(5-250 \mu \mathrm{M})$ and inhibitor (APPI monomer, 0-400 nM; APPI dimer, 0$96 \mathrm{nM}$ ) in a Synergy 2 Multi-Detection Microplate Reader (BioTek, VT, USA). Mesotrypsin buffer $(296 \mu \mathrm{L}), \mathrm{Z}-\mathrm{GPR}-\mathrm{pNA}(8 \mu \mathrm{L})$, and diluted APPI monomer/dimer $(8 \mu \mathrm{L})$ were mixed and equilibrated in a $96-$-well microplate (Greiner, Kremsmünster, Austria) prior to the addition of mesotrypsin ( $8 \mu \mathrm{L}$ from $10 \mathrm{nM}$ stock). Reactions were followed spectroscopically for $5 \mathrm{~min}$, and initial rates were determined from the increase in absorbance caused by the release of p-nitroaniline. Data were globally fitted by multiple regression to Eq. 9, the classic competitive inhibition equation, using Prism (GraphPad Software, San Diego, CA).

$u=\frac{k_{c a t}[E]_{0}[S]}{K_{m}\left(1+[I] / K_{i}\right)+[S]}$

Where $u$ is the velocity of product formation at the start of the reaction; $K_{m}$ (the Michaelis-Menten constant) and $k_{c a t}$ are the kinetic parameters for substrate hydrolysis; $[E]_{0}$ and $[I]$ are the total concentrations of enzyme and inhibitor, respectively, and [S] is the initial substrate concentration. The reactions were performed with excess APPI dimer and monomer concentrations ( $\geq 20$ times the mesotrypsin concentration), and therefore any reduction of inhibitor concentration upon binding was negligible. Reported inhibition constants are average values obtained from three independent experiments, and are expressed as mean $\pm \mathrm{SD}$.

Acknowledgments: The authors thank Dr. Itay Cohen for the technical assistance and advice. This work was supported by the Worldwide Cancer Research (grant number 20-0238) and the European Research Council Proof of Concept grant (grant number 875197) to N.P., and the United States - Israel Binational Science Foundation (grant number 2019303) to N.P and E.S.R, and the Israel Science Foundation (ISF) (grant number $1004 / 20)$ to D.B.L.

Conflict of interest: The authors declare that they have no conflicts of interest with the contents of this article.

Author contributions: S.L.H. and N.P. designed the research; S.L.H. performed the research; S.L.H, Y.S., R.B., D.B.L., and N.P. analyzed data; E.S.R. provided mesotrypsin; S.L.H and N.P. wrote the paper. All authors edited the manuscript and approved the final version. 


\section{References}

1. Ehrenstein MR \& Notley CA (2010) The importance of natural IgM: scavenger, protector and regulator. Nat Rev Immunol 10(11):778-786.

2. Acuner Ozbabacan SE, Engin HB, Gursoy A, \& Keskin O (2011) Transient protein-protein interactions. Protein Eng Des Sel 24(9):635-648.

3. Levine PM, Imberg K, Garabedian MJ, \& Kirshenbaum K (2012) Multivalent peptidomimetic conjugates: a versatile platform for modulating androgen receptor activity. J Am Chem Soc 134(16):6912-6915.

4. Keskin O, Gursoy A, Ma B, \& Nussinov R (2008) Principles of protein-protein interactions: what are the preferred ways for proteins to interact? Chem Rev 108(4):1225-1244.

5. Mammen M, Choi SK, \& Whitesides GM (1998) Polyvalent Interactions in Biological Systems: Implications for Design and Use of Multivalent Ligands and Inhibitors. Angew Chem Int Ed Engl 37(20):2754-2794.

6. Jones S \& Thornton JM (1996) Principles of protein-protein interactions. Proc Natl Acad Sci U S A 93(1):13-20.

7. Schuchardt BJ, et al. (2014) Ligand binding to WW tandem domains of YAP2 transcriptional regulator is under negative cooperativity. The FEBS journal 281(24):5532-5551.

8. Kiessling LL, Gestwicki JE, \& Strong LE (2000) Synthetic multivalent ligands in the exploration of cell-surface interactions. Curr Opin Chem Biol 4(6):696703.

9. Kiessling LL, Gestwicki JE, \& Strong LE (2006) Synthetic multivalent ligands as probes of signal transduction. Angew Chem Int Ed Engl 45(15):2348-2368.

10. Zur Y, et al. (2017) Engineering a monomeric variant of macrophage colonystimulating factor (M-CSF) that antagonizes the c-FMS receptor. The Biochemical journal 474(15):2601-2617.

11. Varner CT, Rosen T, Martin JT, \& Kane RS (2015) Recent advances in engineering polyvalent biological interactions. Biomacromolecules 16(1):4355.

12. Chen H \& Privalsky ML (1995) Cooperative formation of high-order oligomers by retinoid X receptors: an unexpected mode of DNA recognition. Proc Natl Acad Sci U S A 92(2):422-426.

13. Mammen M, Dahmann G, \& Whitesides GM (1995) Effective inhibitors of hemagglutination by influenza virus synthesized from polymers having active ester groups. Insight into mechanism of inhibition. J Med Chem 38(21):41794190.

14. Lee BW, et al. (2010) Strongly binding cell-adhesive polypeptides of programmable valencies .Angew Chem Int Ed Engl 49(11):1971-1975.

15. Kiessling LL \& Pohl NL (1996) Strength in numbers: non-natural polyvalent carbohydrate derivatives. Chem Biol 3(2):71-77.

16. Chittasupho C (2012) Multivalent ligand: design principle for targeted therapeutic delivery approach. Ther Deliv 3(10):1171-1187.

17. Lees WJ, Spaltenstein A, Kingery-Wood JE, \& Whitesides GM (1994) Polyacrylamides bearing pendant alpha-sialoside groups strongly inhibit agglutination of erythrocytes by influenza A virus: multivalency and steric stabilization of particulate biological systems. J Med Chem 37(20):3419-3433. 
18. Jorgensen HF, Adie K, Chaubert P, \& Bird AP (2006) Engineering a highaffinity methyl-CpG-binding protein. Nucleic Acids Res 34(13):e96.

19. Tjandra KC \& Thordarson P (2019) Multivalency in Drug Delivery-When Is It Too Much of a Good Thing? Bioconjug Chem 30(3):503-514.

20. Szmola R, Kukor Z, \& Sahin-Toth M (2003) Human mesotrypsin is a unique digestive protease specialized for the degradation of trypsin inhibitors .The Journal of biological chemistry 278(49):48580-48589.

21. Rinderknecht H, Renner IG, Abramson SB, \& Carmack C (1984) Mesotrypsin: a new inhibitor-resistant protease from a zymogen in human pancreatic tissue and fluid. Gastroenterology 86(4):681-692.

22. Sahin-Toth M (2005) Human mesotrypsin defies natural trypsin inhibitors: from passive resistance to active destruction. Protein and peptide letters 12(5):457464.

23. Salameh MA, Soares AS, Alloy A, \& Radisky ES (2012) Presence versus absence of hydrogen bond donor Tyr-39 influences interactions of cationic trypsin and mesotrypsin with protein protease inhibitors. Protein Sci 21(8):1103-1112.

24. Alloy AP, et al. (2015) Mesotrypsin Has Evolved Four Unique Residues to Cleave Trypsin Inhibitors as Substrates. The Journal of biological chemistry 290(35):21523-21535.

25. Pendlebury D, et al. (2014) Sequence and conformational specificity in substrate recognition: several human Kunitz protease inhibitor domains are specific substrates of mesotrypsin. The Journal of biological chemistry 289(47):32783-32797.

26. Kassell B, Radicevic M, Ansfield MJ, \& Laskowski M, Sr. (1965) The Basic Trypsin Inhibitor of Bovine Pancreas. Iv. The Linear Sequence of the 58 Amino Acids. Biochem Biophys Res Commun 18:255-258.

27. Salameh MA, et al. (2010) Determinants of affinity and proteolytic stability in interactions of Kunitz family protease inhibitors with mesotrypsin. The Journal of biological chemistry 285(47):36884-36896.

28. Salameh MA, et al. (2010) The amyloid precursor protein/protease nexin 2 Kunitz inhibitor domain is a highly specific substrate of mesotrypsin. The Journal of biological chemistry 285(3):1939-1949.

29. Kunitz M \& Northrop JH (1936) Isolation from Beef Pancreas of Crystalline Trypsinogen, Trypsin, a Trypsin Inhibitor, and an Inhibitor-Trypsin Compound. The Journal of general physiology 19(6):991-1007.

30. Bisswanger H (2002) Enzyme kinetics : principles and methods (Wiley-VCH, Weinheim Germany) pp xiv, 255 p.

31. Cohen I, et al. (2016) Combinatorial protein engineering of proteolytically resistant mesotrypsin inhibitors as candidates for cancer therapy. The Biochemical journal 473(10):1329-1341.

32. Xu Y, Carr PD, Guss JM, \& Ollis DL (1998) The crystal structure of bikunin from the inter-alpha-inhibitor complex: a serine protease inhibitor with two Kunitz domains. J Mol Biol 276(5):955-966.

33. Krishnamurthy VM, Estrofi LA, \& Whitesides GM (2006) Multivalency in Ligand Design.

34. Handl HL, et al. (2004) Hitting multiple targets with multimeric ligands. Expert Opin Ther Targets 8(6):565-586.

35. Hadzhieva $\mathrm{M}$, et al. (2017) Impact of Antigen Density on the Binding Mechanism of IgG Antibodies. Sci Rep 7(1):3767. 
36. Chen X, Zaro JL, \& Shen WC (2013) Fusion protein linkers: property, design and functionality. Adv Drug Deliv Rev 65(10):1357-1369.

37. Hogg PJ (2003) Disulfide bonds as switches for protein function. Trends in biochemical sciences 28(4):210-214.

38. Laskowski M, Jr. \& Kato I (1980) Protein inhibitors of proteinases. Annu Rev Biochem 49:593-62.6

39. Lukatsky DB \& Frenkel D (2004) Phase behavior and selectivity of DNAlinked nanoparticle assemblies. Phys Rev Lett 92(6):068302.

40. Lukatsky DB \& Frenkel D (2005) Surface and bulk dissolution properties, and selectivity of DNA-linked nanoparticle assemblies. J Chem Phys 122(21):214904.

41. Silacci M, et al. (2014) Linker length matters, fynomer-Fc fusion with an optimized linker displaying picomolar IL-17A inhibition potency. The Journal of biological chemistry 289(20):14392-14398.

42. Wolff EA, Schreiber GJ, Cosand WL, \& Raff HV (1993) Monoclonal antibody homodimers: enhanced antitumor activity in nude mice. Cancer Res 53(11):2560-2565.

43. Papo N, Silverman AP, Lahti JL, \& Cochran JR (2011) Antagonistic VEGF variants engineered to simultaneously bind to and inhibit VEGFR2 and alphavbeta3 integrin. Proc Natl Acad Sci U S A 108(34):14067-14072.

44. Salameh MA, Soares AS, Hockla A, \& Radisky ES (2008) Structural basis for accelerated cleavage of bovine pancreatic trypsin inhibitor (BPTI) by human mesotrypsin. The Journal of biological chemistry 283(7):4115-4123.

45. Salameh MA, Soares AS, Hockla A, Radisky DC, \& Radisky ES (2011) The $\mathrm{P}(2)^{\prime}$ residue is a key determinant of mesotrypsin specificity: engineering a high-affinity inhibitor with anticancer activity. The Biochemical journal 440(1):95-105.

46. Chase T, Jr. \& Shaw E (1967) p-Nitrophenyl-p'-guanidinobenzoate HCl: a new active site titrant for trypsin. Biochemical and biophysical research communications 29(4):508-514. 